\title{
Cavitation erosion behaviour of the steel plate of a scroll expander system
}

\author{
I. Tzanakis ${ }^{1}$, A. Georgoulas ${ }^{2}$, M. Hadfield ${ }^{1} \&$ N. Kotsovinos ${ }^{2}$ \\ ${ }^{I}$ Sustainable Design Research Centre, School of Design, \\ Engineering and Computing, Bournemouth University, UK \\ ${ }^{2}$ Laboratory of Hydraulics and Hydraulic Structures, \\ Fluid Mechanics Division, Department of Civil Engineering, \\ Democritus University of Thrace, Greece
}

\begin{abstract}
A steel plate is one of the critical components of a scroll expander system that usually experiences cavitation in service. An experimental study is conducted to study the behaviour of the scroll's steel plate subjected to cavitation erosion. For this purpose an ultrasonic transducer is utilised to produce cavitation bubbles. Micrographs of damaged surfaces were observed, showing the nature of the cavitation mechanism and the morphology alterations across the steel sample. Experimental results are explained in terms of the cavitation erosion rates, roughness profile, accumulated strain energy, and hardness of the matrix.

Keywords: ultrasonic cavitation, erosion, bubbles, refrigerant, high carbon steel.
\end{abstract}

\section{Introduction}

Scroll expander systems are facing cavitation wear problems because of their long operational period, their complicated geometry, their mechanical design and their high-speed working fluids [1].

Cavitation is a dynamic phenomenon known as a repeated formation and violent collapse of cavitation bubbles in a liquid caused by the instantaneous transition of liquid phase to gas phase due to rapid fluctuations of local pressure. However in applications like scroll expander systems where pressure fluctuations are at critical levels and the refrigerant working fluid is in a vapour phase, cavitation can be initiated by the localised phase change from vapour to liquid $[2,3]$. Cavitation erosion damage is caused by removal of material or plastic 
deformation of a solid surface when it undergoes high pressure impacts during a cavity collapse. The implosion of cavitation bubbles generates large amplitude shock waves and powerful micro-jets resulting to highly localized and transient surface stresses $[4,5]$. The solid boundaries absorb that impact energy, leading to elastic deformation, plastic deformation or fracture [6].

An experimental study based on previous cavitation erosion research outcomes investigates the formation of erosion pits into deep craters [7, 8]. The resistance of the steel plate against cavitation for a prolonged period of time is determined. The cavitation-erosion mechanisms affecting the durability of scroll's steel plate were revealed while interesting correlation between the material properties of the steel plate and the cavitation erosion rates were found.

\section{Experimental methodology}

\subsection{Test rig}

Cavitation erosion tests were utilized in the present study to analyse and evaluate the behaviour of the scroll's steel plate against cavitation. The experiments were carried out based upon the modified ASTM G32-03 standard method described in reference [7]. An ultrasonic vibratory transducer with vibratory frequency of $20 \mathrm{kHz}$ and peak-to-peak vibratory amplitude of $50 \mu \mathrm{m}$ is deployed. The high intensity ultrasound is applied to the liquid medium generating cavitation bubbles. The liquid medium used for cavitation tests was distilled water with physical properties the same as in [8].

The steel samples were mounted on the bottom of a $5 \mathrm{~L}$ experimental tank while the tip of the horn is submerged into distilled water to a distance of $0.5 \mathrm{~mm}$ from the top of the steel sample (figure 1). Cavitation erosion tests were performed over a prolonged period of time within the range of 1 to 8 hours. After the end of each experiment the durability and resistance of the steel samples to cavitation is evaluated by measuring the erosion wear rates using profilometric

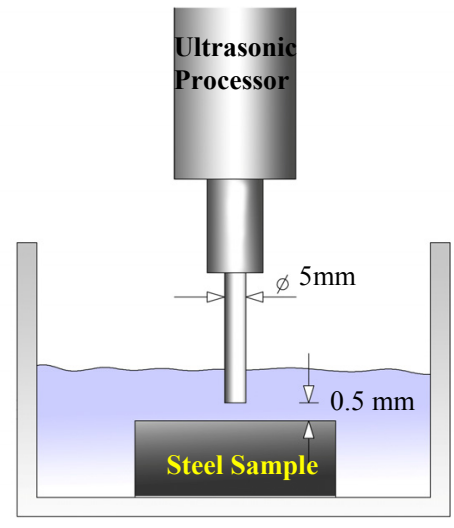

Figure 1: Cavitation experimental test rig. 
techniques. Additionally, after the completion of the cavitation tests, microhardness tests were performed using an $8.825 \mathrm{~N}$ applied load. The correlation between the strain energy, the roughness and the hardness evolution of the steel samples against their cavitation erosion rates is determined.

\subsection{Test samples}

The steel plate of the scroll expander was initially etched in a solution of $2 \%$ Nital in order for their grain structure to be determined. An Optical Emission Spectrometer (OES) was used in distinguishing the samples in their individual chemical elements (Table 1). Thereinafter the scroll plate is machined into appropriate samples shape by a cutting machine. Then the steel samples were implemented on a Bakelite base and polished in a few microns accuracy prior to the test. The steel plate has an initial hardness of 540 (HV) while its materials properties can be found in [6].

Table 1: Chemical analysis of the scroll's steel plate.

\begin{tabular}{|ccccccccc|}
\hline Composition & $\mathbf{C}$ & $\mathbf{M n}$ & $\mathbf{S i}$ & $\mathbf{S}$ & $\mathbf{P}$ & $\mathbf{C u}$ & $\mathbf{N i}$ & $\mathbf{C r}$ \\
$\mathrm{Wt} \%$ & 0.96 & 0.53 & 0.27 & 0.003 & 0.021 & 0.01 & 0.02 & 0.18 \\
\hline
\end{tabular}

\section{Experimental results}

\subsection{Microscopic examination}

Over a prolonged period of time, the incubation pits which were formed in the early stages of the cavitation mechanism can significantly grow by linking up with the nearby pits, cracks and grooves [7-9]. The surface roughness substantially increases accelerating the erosion rate of the materials. The enlargement of the pits is accompanied by additional volume loss. The mechanism of pit growth leads to the formation of deep and wide craters penetrating the sample's surface. These areas were named "ring areas" because of the shape they obtain after the end of the ultrasonic cavitation process.

A series of SEM micrographs in figure 2 shows the eroded surface of the scroll's steel plate at different stages of the cavitation process. The images were taken in each of the selected test intervals in order to get an overview of the attenuation of the pits and their formation into craters. After 1 hour of testing the initial stage of cavitation erosion is shown as pits formation dominates the erosion regime (figure 2(a)). The next stage is after 3 hours of exposure where a crater shape is formed (figure 2(b)). The periphery of the crater is clearly visible. Its core consists of pits which show increased density and size. After 5 hours of testing, cavitation damage is spread to the vicinity areas of the "ring" and significantly erosion damage is observed (figure 2(c)). However, not substantially material loss was noticed as erosion restricted within the upper substrate of the steel surface while the crater depth remained to similar levels as in figure 2(b). Finally, after 8 hours of testing the crater was severely eroded forming a deep valley. The edges of the periphery were very sharp and sloppy 


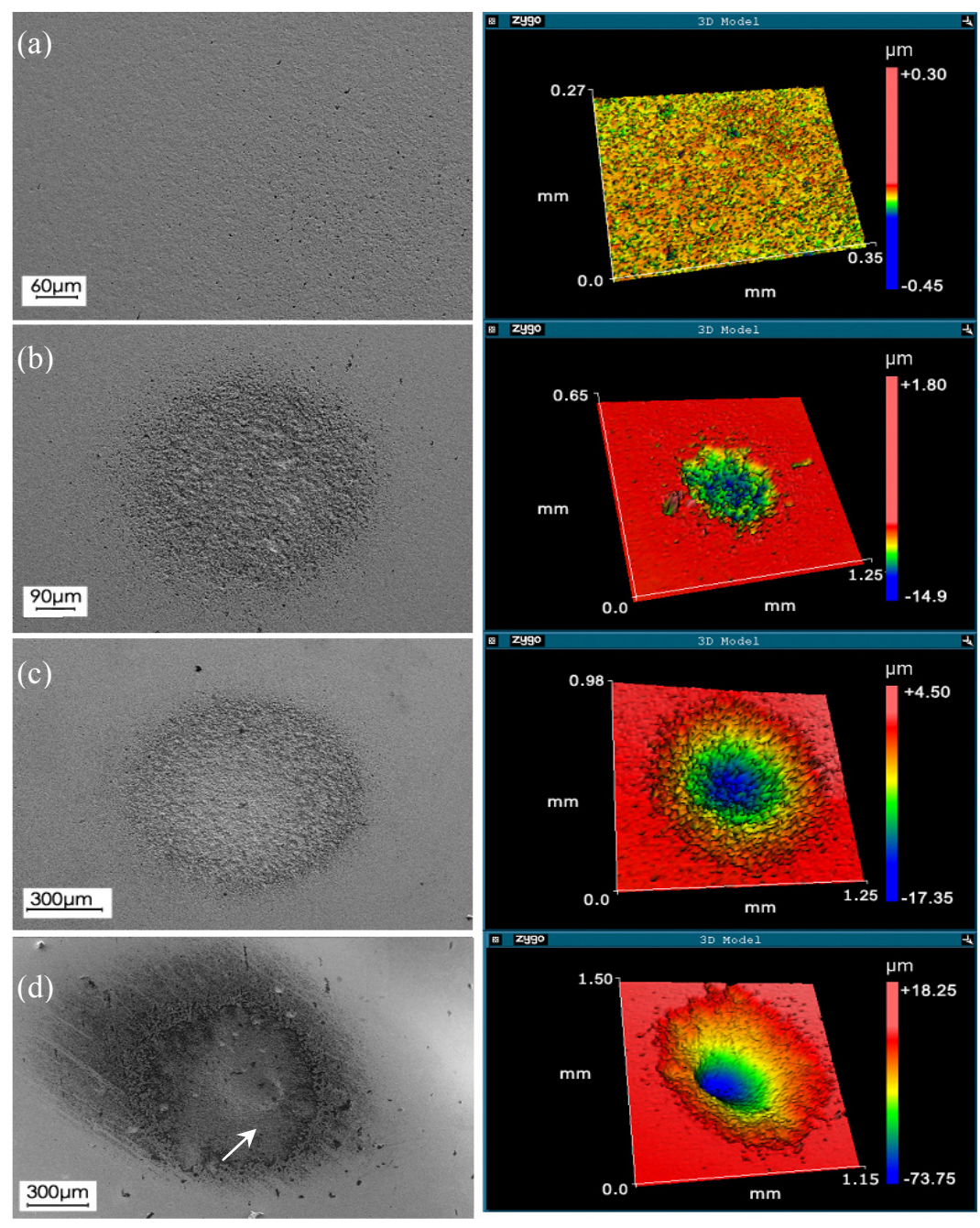

Figure 2: A series of SEM and ZYGO interferometer micrographs showing the evolution of cavitation pits into crater and the volume removed by cavitation erosion impact at different stages (a): 1 hour, (b): 3 hours, (c): 5 hours and (d): 8 hours.

while huge loss of material was noticed. Closer observation demonstrated the increased depth of the crater (figure 2(d), white arrow).

\subsection{Erosion rates}

Measurements were carried out to monitor the volume loss, the roughness profile and the mean depth erosion rate of the steel samples over the different stages of the cavitation erosion tests. 
The erosion loss of material was expressed in terms of the mean depth erosion rate (MDER) (eq. (1)) and the roughness in terms of Ra (eq. (2)) which is the most common roughness parameter. Both of the parameters were defined as it follows:

$$
\operatorname{MDER}(\mu \mathrm{m} / h)=10 \frac{\Delta W}{\rho A \Delta t}
$$

and

$$
R_{a}=\frac{1}{L} \int_{0}^{L}|z(x)| d x
$$

where $\Delta \mathrm{W}$ is the weight loss in $\mathrm{mg}, \Delta \mathrm{t}$ is the test time in hours, $\mathrm{A}$ is the cavitated area of the specimen in $\mathrm{cm}^{2}$, and $\rho$ is the density of the specimen in $\mathrm{g} / \mathrm{cm}^{3}, \mathrm{~L}$ is the length area of the sample surface in $\mu \mathrm{m}$ and $\mathrm{z}$ is the average value of the asperities height in $\mu \mathrm{m}$.

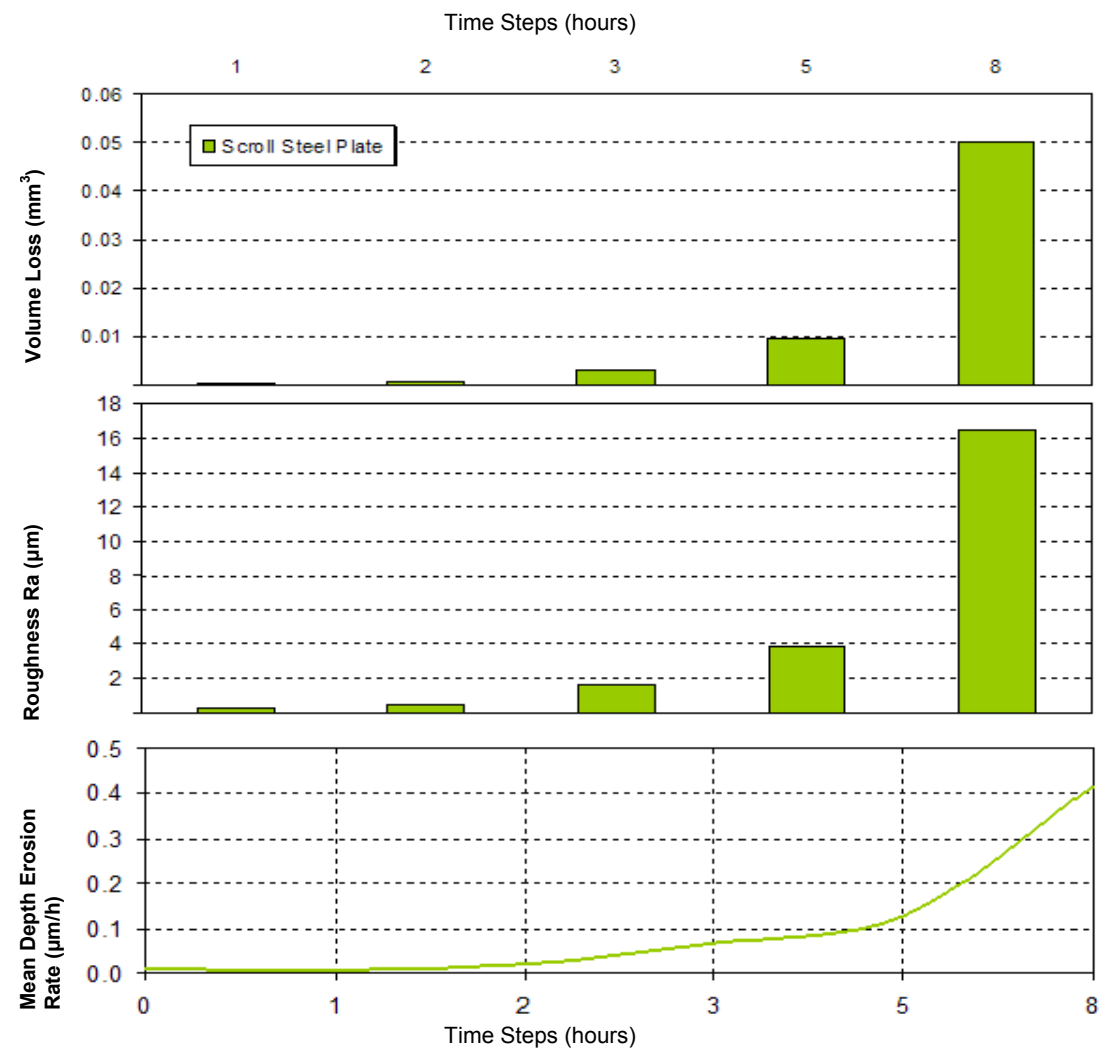

Figure 3: Variation of the volume loss, roughness profile and mean depth erosion rate of the scroll's steel plate for different time steps. 
According to figure 3, all of the parameters are proportionally increasing with time. Specifically, up to 5 hours exposure to cavitation, steel plate exhibits low volume loss (less than $0.01 \mathrm{~mm}^{3}$ ) and MDER (around $0.1 \mu \mathrm{m} / \mathrm{h}$ ) while roughness is changed with a relative low rate. Then, its endurance was dramatically reduced and the erosion rate was steeply increased (more than $0.4 \mu \mathrm{m} / \mathrm{h}$ ). The heat influence, the repeated impact pressure and the interactions between the dislocations and the grain structure cause the material flow and to significantly drop its hardness (figure 4) made it vulnerable to cavitation. Hence, over a prolonged period ( 8 hours) of exposure to cavitation impacts the steel plate of the scroll faced inevitable severe damage.

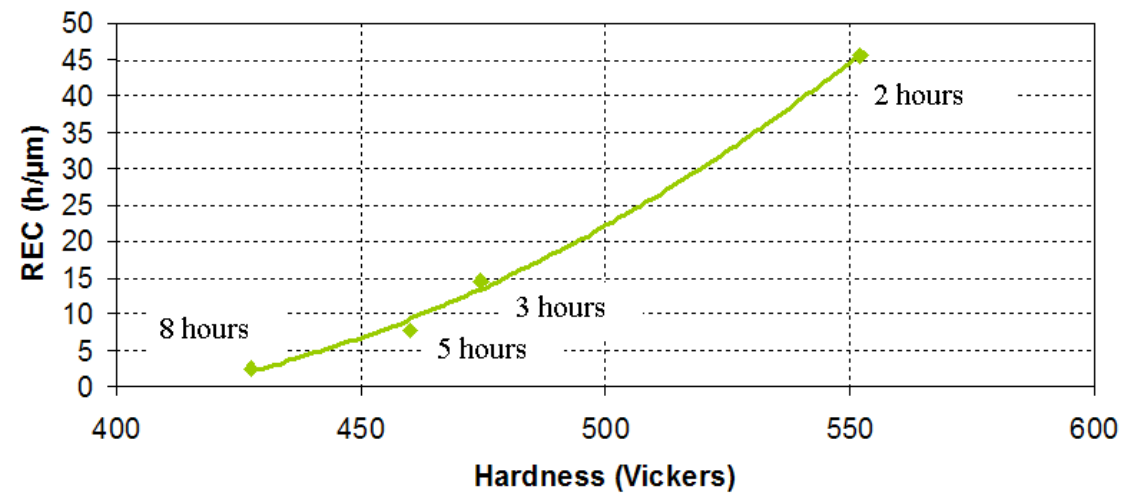

Figure 4: Hardness measurements against erosion resistance of the steel plate for different time steps.

\subsection{Hardness}

The change in hardness can be closely correlated with the erosion resistance (REC) of the steel samples (eq. (3)). Generally, higher resistance implies higher hardness values. The cavitation erosion resistance (REC) is defined as the reciprocal of the mean erosion rate and expressed as follows:

$$
\operatorname{REC}(h / \mu m)=\left(\frac{1}{M D E R}\right)
$$

In every steel sample the hardness alterations depend on the intention and the time duration of the cavitation impacts. If conditions are suitable work hardening on the steel samples is occurs, otherwise rapid damage can occur.

In figure 4 it can be seen that the hardness in monotonically increases with the increment of the erosion resistance of the steel plate. Interestingly for the test duration of 2 hours the hardness of the steel has slightly increased. This can be interpreted by the fact that dislocation movements were possibly restricted, increasing their density in those areas. Consequently, the hardness and the brittleness of the steel were increased. However after 3 hours of test a rapid decrease of the hardness value of about $100 \mathrm{HV}$ is observed implying the 
rapid reduction of its erosion resistance. From that point onwards the high carbon steel plate shows its brittle character, incapable of resisting the massive striking of the micro-jets, lowering its hardness values significantly.

\subsection{Strain energy}

The cavitation erosion mechanism has a fatigue character; hence the damage needs time to be developed. Since the erosion damage is caused by consecutive impacts similar to a fatigue phenomenon, the accumulated strain energy can indicate the critical point where the damage becomes crucial for the steel. Strain energy estimates the rate of the erosion and the resistance of the samples against cavitation. Thus the accumulated strain energy can be expressed by equation 4 .

$$
\Sigma E n=V \Sigma\left(\frac{\sigma^{2}}{2 E}\right)
$$

where $\mathrm{V}$ is the volume loss in $\mathrm{mg}, \sigma$ is the yield strength in $\mathrm{MPa}$ and $\mathrm{E}$ is the Young modulus in GPa.

The dependency of the accumulated strain energy as a function of the crater depth of the steel samples is depicted in figure 5. As shown, there are three stages of the erosion evolution according the strain energy results. The first stage is the inception of cavitation pits until the formation of small craters. In this stage strain energy slightly increases for the first 3 hours of the test. Then for the next 2 hours the crater depth stabilises to a value around $20 \mu \mathrm{m}$ as volume loss is mainly observed from the peripheral areas of the crater rather than at places within the crater (figure 2(c)). Consequently, strain energy rate which is accumulated in a larger area within the mass of the steel increases by about 3 times. When the strain energy exceeds the limit of the $15 \mathrm{~mJ}$ the eroded damage is localised forming deep craters. In that stage a notable volume loss is observed while the erosion rates are significantly accelerated.

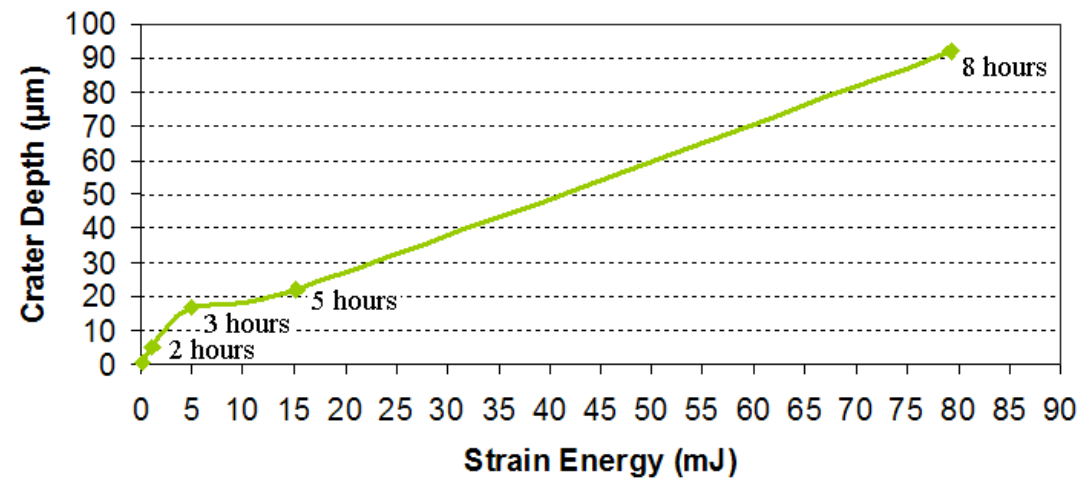

Figure 5: Correlation between the accumulated strain energy and the crater depth of the steel plate for different time steps. 


\section{Summary and conclusions}

This study presents an assessment of the cavitation mechanism within a scroll expander system using a quantitative evaluation of the scroll's steel plate behaviour against cavitation erosion.

The experimental results from the ultrasonic transducer determine the endurance of the steel plate material against cavitation attack. A correlation between the volume loss and the cavitation erosion rate for different time steps of the steel plate material is achieved. Results showed that steel plate exhibits a good performance against cavitation for the first 5 hours of the test. After that time step, repeated impacts of cavitation pulses lead to accelerative breakage of the substrate as it crystal structure cannot sustain the new energy income and the material experiences severe erosion. The evolution of the roughness profile with time is also determined. It is characteristic that roughness steadily increases until the first 5 hours of exposure to cavitation with a relatively low rate, while after that point the proposed rate shows a rapid increment by approximately 4 times accelerating the erosion rate of the steel samples.

Additionally, the cavitation-erosion mechanisms affecting the durability of scroll's steel plate are explicitly depending on its hardness and the ability of the material to accumulate strain energy. Hardness is proved to be one of the key factors in increasing the lifetime of the steel plate as it is directly correlated with the erosion resistance of the material. Moreover, it is deduced that the eroded damage was localized when accumulated strain energy of more than $15 \mathrm{~mJ}$ is applied to the sample by repeated impact.

For future investigation it is necessary to further extend the content of the present study, conducting experimental measurements among similar industrial steel grades, aiming to identify a higher cavitation-resistant steel material over a prolonged period of time.

\section{References}

[1] I. Tzanakis, M. Hadfield, B. Thomas, S. M. Noya, S. Austen and I. Hensaw. 2012 "Future Perspectives of Sustainable Tribology". Renewable and Sustainable Energy Reviews, Volume 16, Issue 6, (4126-4140).

[2] I. Tzanakis, M. Hadfield, Z. Khan. 2009. "Durability of domestic scroll compressor systems". WIT Transactions on Engineering Sciences, Volume $62(229-240)$.

[3] I. Tzanakis, M. Hadfield, T. Georgoulas, N. Kotsovinos. 2010. "Cavitation damage observations within scroll expander lubrication systems". WIT Transactions on Engineering Sciences, Volume 66 (261-272).

[4] A. Krella, A. Czyzniewski. 2007. "Influence of the substrate hardness on the cavitation erosion resistance of TiN coating". Wear, 263 (395-401).

[5] P.R. Williams, P.M. Williams, S.W.J. Brown. 1997. "A technique for studying liquid jets by cavitation bubble collapse under shockwaves, near a free surface". Journal of Non-Newtonian Fluid Mechanics, 72 (101-110). 
[6] B.S Mann. 2000. "High-energy particle impact wear resistance of hard coatings and their application in hydroturbines". Wear, 237 (140-146).

[7] I. Tzanakis, N. Garland and M. Hadfield. 2011. "Cavitation damage incubation with typical fluids applied to a scroll expander system". Tribology International, Volume 44, Issue 12, (1668-1678).

[8] I. Tzanakis and M. Hadfield. 2011. "Observations of acoustically generated cavitation bubbles within typical fluids applied to a scroll expander lubrication system". Experimental Thermal and Fluid Science, Volume 35, Issue 8, (1544-1554).

[9] B. Karunamurthy, M. Hadfield, C. Vieillard, G. Morales. 2010. "Cavitation erosion in silicon nitride: experimental investigations on the mechanism of material degradation" Tribology International, 43 (2251-7). 